\title{
Psychiatric Medication Users by Age and Sex in the United States, 1999-2018
}

\author{
Michael E. Johansen, MD, MS
}

Purpose: This study aimed to determine the rates of psychiatric medication users in the United States between 1999 to 2018 for different medication categories by age and sex.

Methods: The 1999 to 2002, 2006 to 2009, and 2015 to 2018 Medical Expenditure Panel Surveys (MEPS) were used for the analysis. All individuals with a valid age were included. Any antidepressant, benzodiazepine, attention deficit hyperactivity disease (ADHD) medication, antipsychotics, and mood stabilizer report use was defined as a medication user. Separate multivariable logistic regression predicted medication users by age with restricted cubic splines by sex, medication category, and year category (1999 to 2002, 2006 to 2009 , and 2015 to 2018). In addition, the rate of prescribing to males and females at different ages was determined for medication categories.

Results: Rates of any psychiatric medication users increased during the study period. Females had higher rates of medication users around 20 years of age. Rates of antidepressant users increased over time and were higher for females after earlier adolescence. Rates of benzodiazepine users were higher for females, increased after 1999 to 2002, and had consistent patterns of use over time. Antipsychotic and mood stabilizer user rates were lower than other categories. Adolescent antipsychotic users markedly decreased between 2006 to 2009 and 2015 to 2018. Rates of ADHD medication users increased over time, particularly among younger adults between 25 and 50 years of age.

Conclusion: The rates of individuals reporting the use of any psychiatric medications increased over the last few decades. Rates and patterns of medication users have large variations by medication category, age, and sex, but these patterns of use were stable for most medication categories. ( $\mathrm{J}$ Am Board Fam Med 2021;34:732-740.)

Keywords: Attention Deficit Disorder With Hyperactivity, Benzodiazepines, Health Services Research, Logistic Regression, Mental Health Services, Primary Health Care, Psychiatry, Psychopharmacology, Psychotropic Drugs, Surveys and Questionnaires

\section{Introduction}

Previous studies have investigated the use of psychiatric medications in the United States. ${ }^{1-8}$ However, these studies did not clearly identify changes in medication use over time by age and sex. Therefore, the proportion of the population by age and sex that used any psychiatric medication and by classes of psychiatric medication between 1999 to 2018 was investigated.

This article was externally peer reviewed.

Submitted 17 November 2020; revised 18 January 2021; accepted 18 January 2021.

(MEJ).

From the Grant Family Medicine, Ohio Health, Columbus

Funding: None.

Conflict of interest: None.

Corresponding author: Michael Johansen, MD, MS, 290 East Town Street, Suite 670, Columbus, OH 43215, Phone: 614-788-5400 (E-mail: mikejoha3@gmail.com).

\section{Methods}

This descriptive study included individuals with an applicable age from the 1999 to 2002, 2006 to 2009, and 2015 to 2018 Medical Expenditure Panel Survey (MEPS) ${ }^{9}$ were included in the analysis. The MEPS is sponsored by the Agency of Health care Research and Quality (AHRQ) and is nationally representative of the noninstitutionalized population of the United States. The survey is conducted through 5 interviews over 2 years on 2 overlapping cohorts and includes a complex array of methods of collecting and confirming utilization data. ${ }^{8}$

Psychiatric medications were identified by name and grouped into antidepressants (selective serotonin reuptake inhibitors, serotonin-norepinephrine reuptake inhibitors, tricyclic antidepressants, trazodone, nefazodone, vilazodone, bupropion, and 
mirtazapine), benzodiazepines (including zolpidem, eszopiclone, and temazepam), antipsychotics (both typical and atypical), mood stabilizers (lithium, carbamazepine, oxcarbazepine, valproic acid, and lamotrigine), attention deficit hyperactivity disorder (ADHD) medications (stimulants and atomoxetine), and buspirone. A medication user was defined as any reported fill of a medication in the defined classes. Buspirone was only included in the any psychiatric medication analysis.

The proportion of individuals using any psychiatric medication, any antidepressant, any benzodiazepine, any antipsychotic, any mood stabilizer, and any ADHD medication during a year was determined using multivariable logistic regressions by sex and year grouping (1999 to 2002, 2006 to 2009, and 2015 to 2018). Restricted cubic splines with 5 knots were used for psychiatric medications, antidepressants, benzodiazepines, and ADHD medication and 4 knots were used for antipsychotics and mood stabilizers. The "adjustrcspline" command was then utilized to construct post-regression estimates.

The rate ratio of female-to-male users was determined for each drug category by dividing the rates for the 6 categories within each decade of life. Given small sample sizes in certain age ranges, some medication classes were grouped into larger age ranges. Antipsychotics, mood stabilizers, and benzodiazepines were grouped as 0 to 19 years of age. ADHD medications were top coded at 50 or more years of age. Mood stabilizers and antipsychotics were top coded as 60 or more years of age.

Analyses were conducted using complex survey weighting in STATA version 15 (STATA Corporation). The Ohio Health Institutional Review Board ruled the study exempt.

\section{Results}

The sample included 386,977 individuals for the selected years between 1999 to 2018. Overall, the proportion of individuals reporting any psychiatric medication increased from $10.7 \%$ (95\% confidence interval (CI), 10.5 to 11.0 ) in 1999 to 2002 to $15.6 \%$ (95\%CI, 15.1 to 16.0 ) in 2015 to 2018. Rates of medication users of any psychiatric medication varied by age and sex. Rates of males who used a medication were higher before 20 years of age, but afterward, females were more likely to be medication users. The curves shifted upward following 1999 to 2002. Otherwise, small changes were seen in use patterns by sex. For instance, the proportion of females between 15 to 30 years and

Figure 1. Proportion of Individuals Reporting Any Psychiatric Medication by Age, Sex, and Time Period. The figure represents the proportion of individuals reporting any psychiatric medication during a year. Separate multivariable logistic regression used by sex and time period. Restricted cubic splines were utilized to model age. The dashed lines represented $95 \%$ confidence intervals.
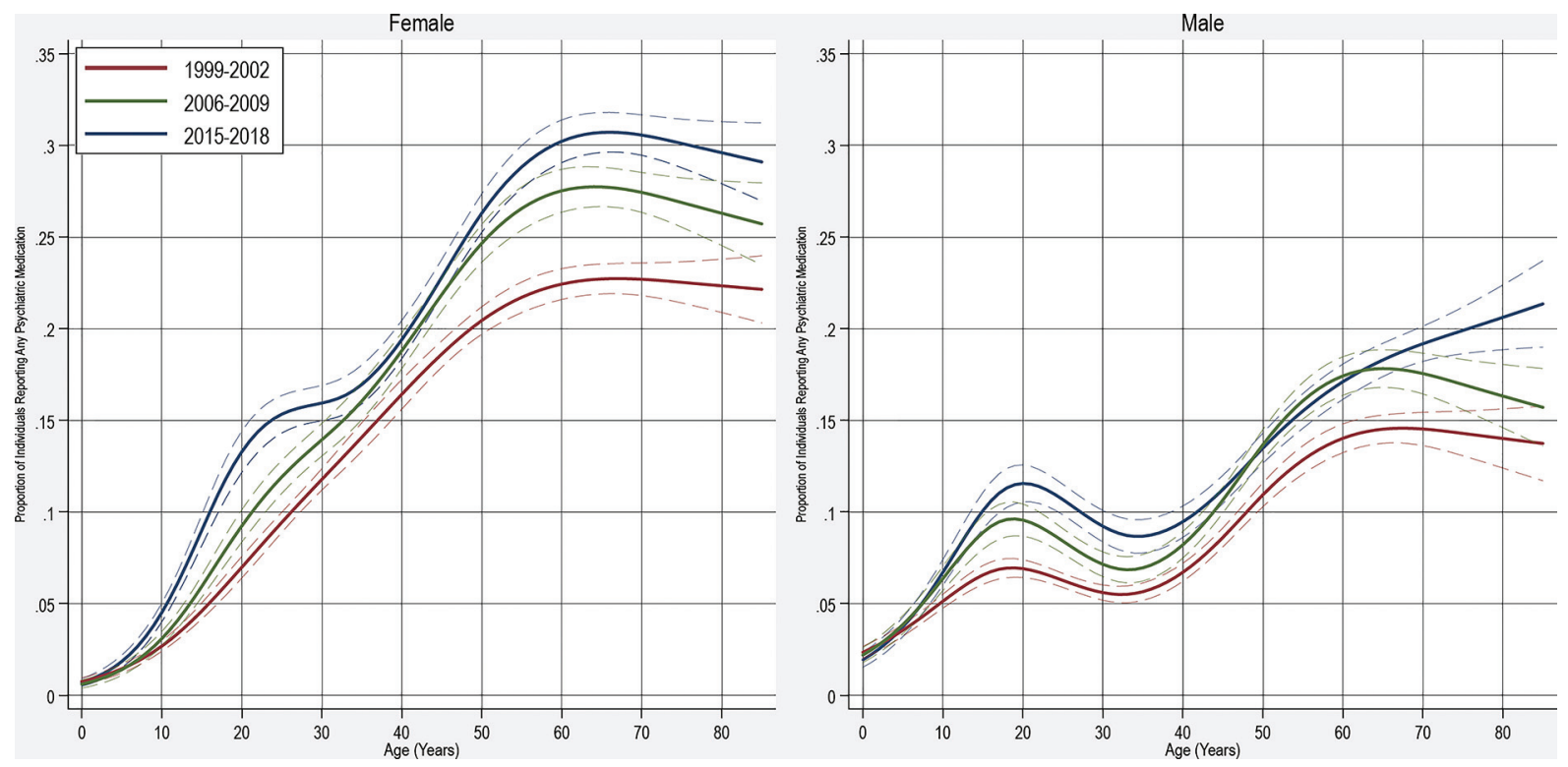
$>60$ years had increases in rates of use between 2006 to 2009 and 2015 to 2018 (Figure 1).

\section{Anti-depressants}

Antidepressant users during a year increased from $7.3 \%$ (95\%CI, 7.1 to 7.5 ) in 1999 to 2002 to $10.9 \%$ (95\%CI, 10.5 to 11.3 ) in 2015 to 2018. Females more often reported antidepressant use than males by around 10 years of age. The highest rates of users were females aged between 55 to 75 years. Rates of male antidepressant users continued to increase with increasing age in 2015 to 2018, while rates attenuated for females after 75 years of age for all time periods. The female and male rate of users increased between 1999 to 2002 and 2015 to 2018 particularly between those 15 to 30 years and over 40 years (Figure 2).

\section{Benzodiazepines}

Rates of users were consistently higher for females compared with males. The rate increased at a younger age for females than males with the largest relative difference in the rate of users at around 30 years of age. Rates of users clearly increased with age for both sexes with similar patterns in rates in all 4-year categories. Rates of users increased between 1999 to 2002 and 2006 to 2009, but notably less so between 2006 to 2009 and 2015 to 2018. There was no strong evidence of a decline in users among individuals aged $>75$ years (Figure 3 ).

\section{Antipsychotics}

Rates of antipsychotic users were notably lower than antidepressants and benzodiazepines. The patterns of use also changed over the 3 time periods. For instance, among young males ( $<25$ years), the rates of use were low from 1999 to 2002, then notably increased during 2006 to 2009 before a dramatic reduction by 2015 to 2018. Females between 25 to 65 years had a notable increase in rates of use following 1999 to 2002. No notable changes in rates of use were identified among individuals older than 75 years (Figure 4).

\section{Mood stabilizers}

Rates of users were similar to antipsychotics for both men and women. Rates appeared to have less change than many other medication categories. Rates of users did not seem to have had much change in males or females $<25$ years or $>60$ years. However, rates of use were increased among individuals between 35 to 55 years. Rates of mood stabilizer users were similar between females and males at most age ranges, but small differences were unlikely to be identified due to limited sample size (Figure 5).

Figure 2. Proportion of Individuals Reporting Any Anti-Depressant Medication by Age, Sex, and Time Period. The figure represents the proportion of individuals reporting any antidepressant medication during a year. Separate multivariable logistic regression used by sex and time period. Restricted cubic splines were utilized to model age. The dashed lines represented $95 \%$ confidence intervals.
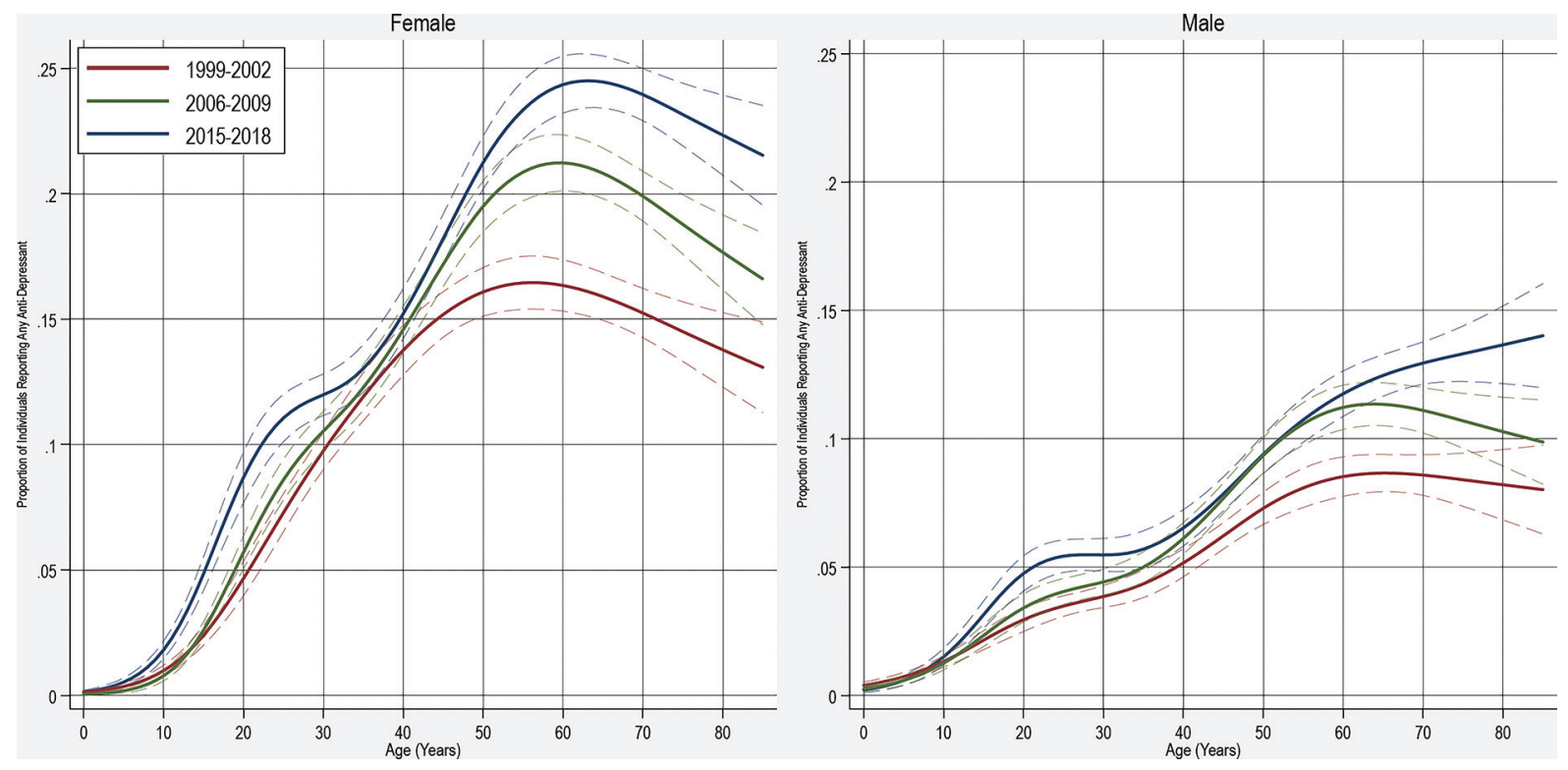
Figure 3. Proportion of Individuals Reporting Any Benzodiazepine by Age, Sex, and Time Period. The figure represents the proportion of individuals reporting any benzodiazepine during a year. Separate multivariable logistic regression used by sex and time period. Restricted cubic splines were utilized to model age. The dashed lines represented $95 \%$ confidence intervals.

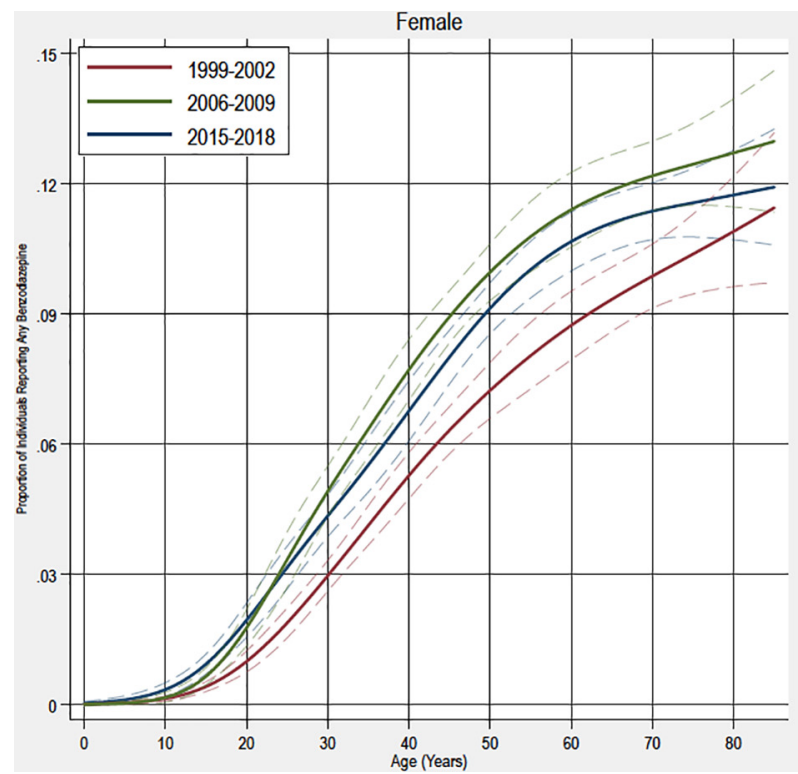

\section{ADHD medications}

Rates of ADHD medication users were higher among males than females in individuals younger than 20 years with the differences by sex being

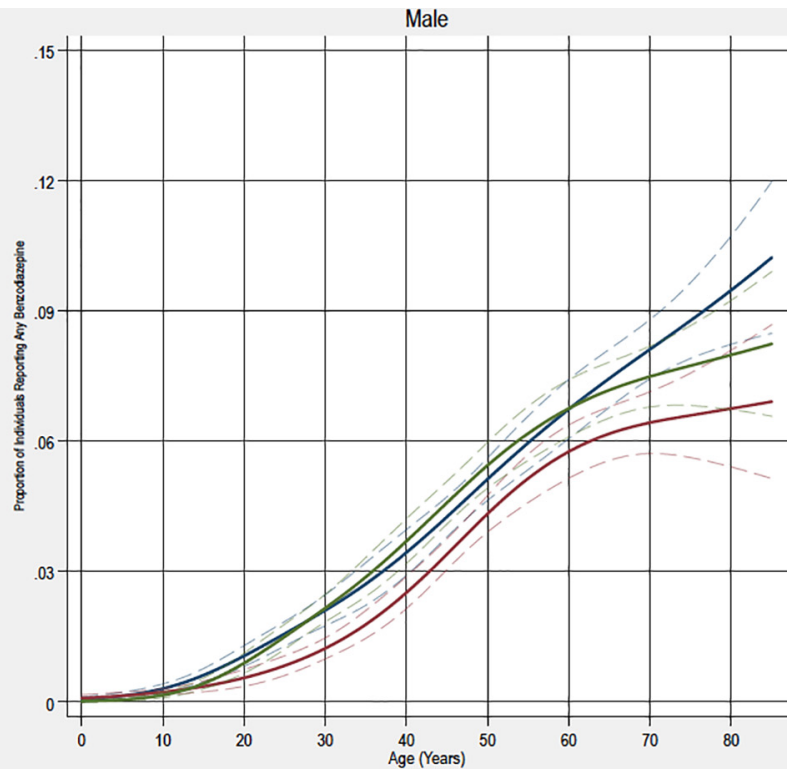

notably less after that age range in 2015 to 2018. Among females, rates increased through the 3 time periods. Among males, there was a notable increase among individuals <20 years between 1999 to 2002

Figure 4. Proportion of Individuals Reporting Any Antipsychotic Medication by Age, Sex, and Time Period. The figure represents the proportion of individuals reporting any antipsychotic medication during a year. Separate multivariable logistic regression used by sex and time period. Restricted cubic splines were utilized to model age. The dashed lines represented $95 \%$ confidence intervals.
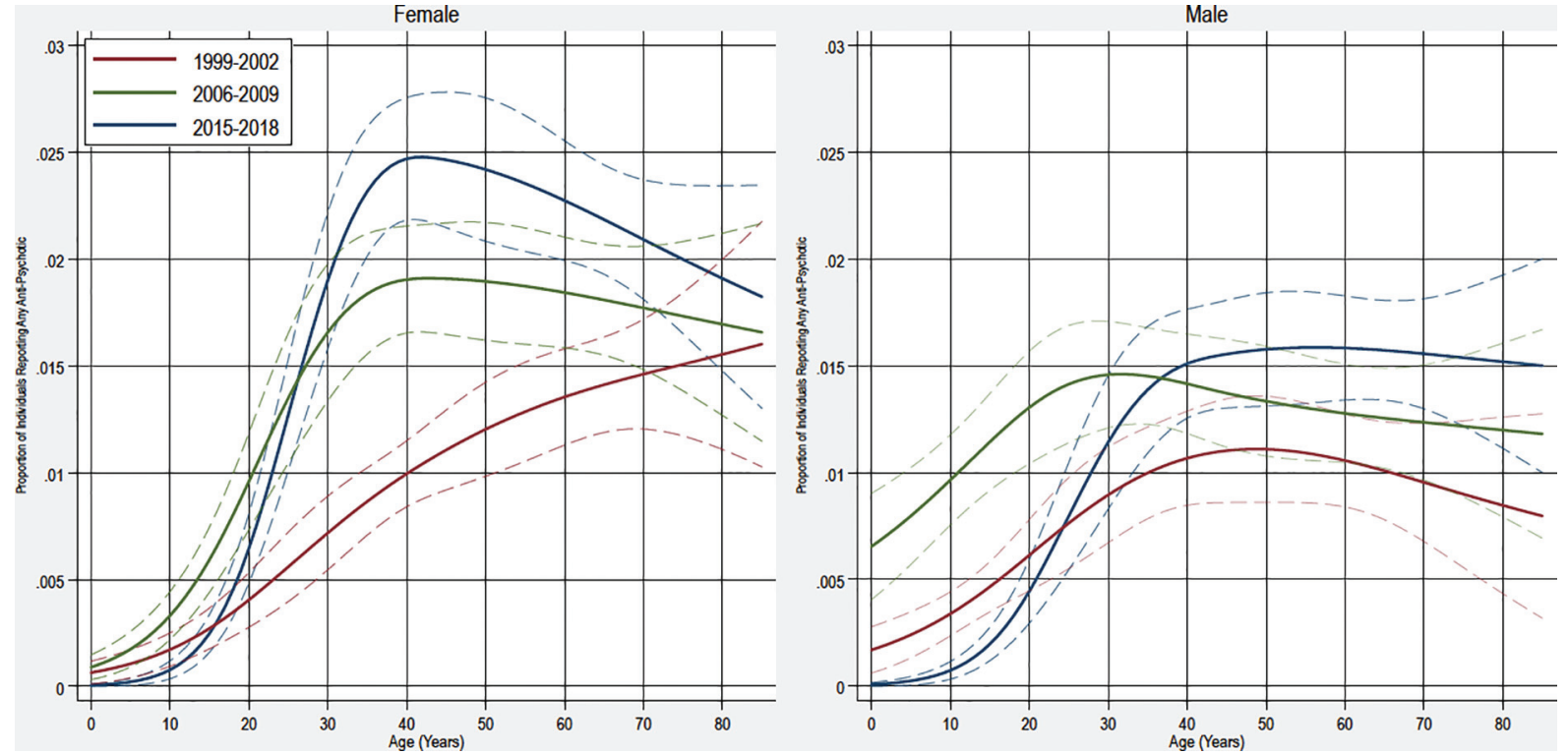
Figure 5. Proportion of Individuals Reporting Any Mood Stabilizer by Age, Sex, and Time Period. The figure represents the proportion of individuals reporting any mood stabilizer during a year. Separate multivariable logistic regression used by sex and time period. Restricted cubic splines were utilized to model age. The dashed lines represented 95\% confidence intervals.
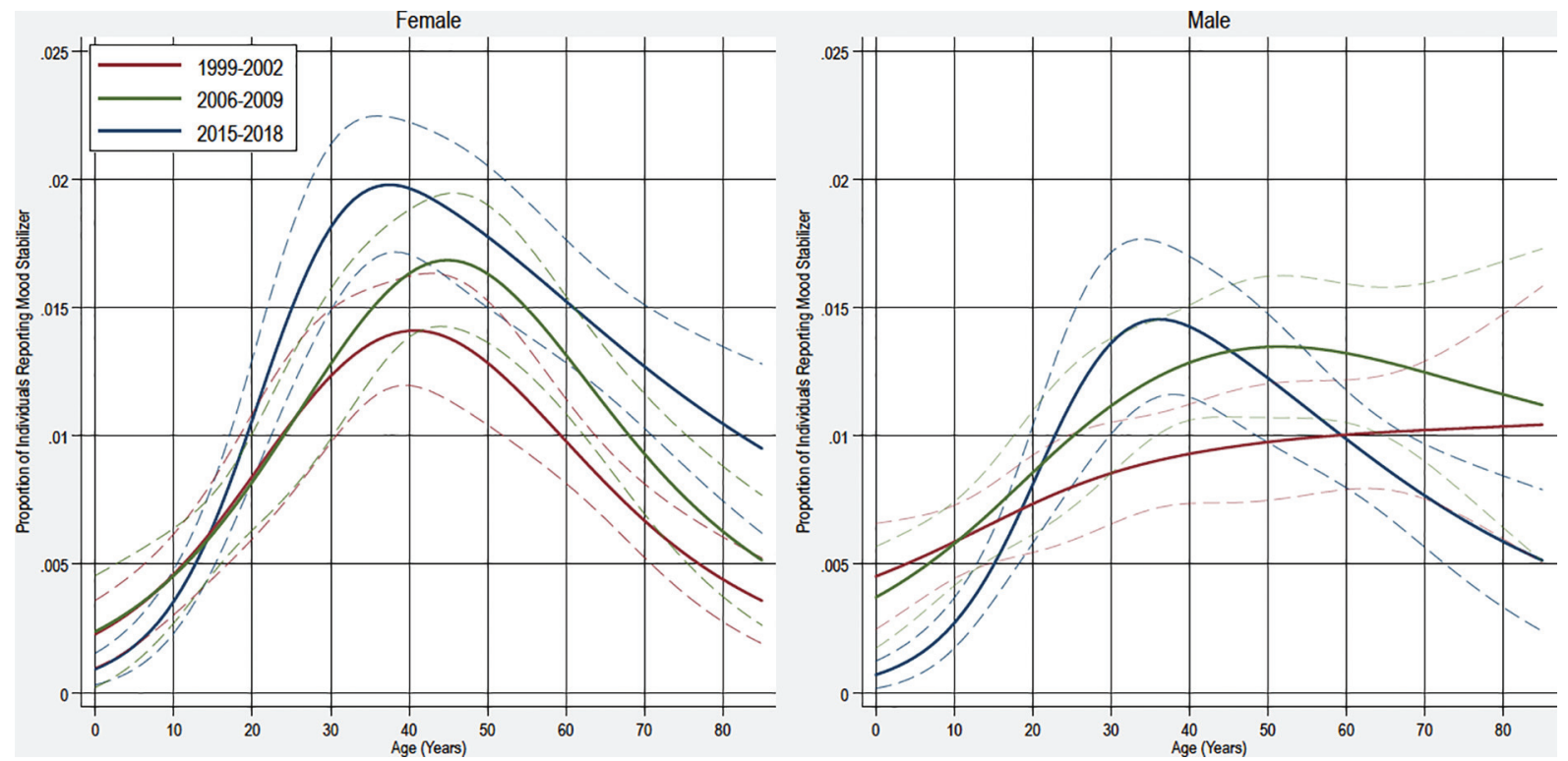

and 2006 to 2009 and for those 25 to 50 years between 2006 to 2009 and 2015 to 2018 (Figure 6).

\section{Medication class comparisons}

Rates of medication users by class from 2015 to 2018 were analyzed separately for males and females to allow easier comparison between different medication classes (Figure 7). Males had higher rates of any psychiatric medication users at younger ages related to the predominance of ADHD medications in that age range. Rates of antidepressant users were higher in females starting in the teens and persisting through older ages. The rate of benzodiazepine users was higher among females from 20 to 79 years, while rates of antipsychotic users were higher among females between 30 to 59 years. There was no convincing evidence of differences in the rate of mood stabilizer users by sex at any age. While males were more likely to be ADHD medication users at $<20$ years of age, there was not a notable difference after 20 years of age (Figure 8).

\section{Discussion}

The proportion of the population who reported any psychiatric medication varied markedly by age and sex, but the proportion of medication users increased between 1999 to 2002 and 2015 to 2018 while patterns remained relatively stable. Between 2015 to 2018 more than $25 \%$ of females older than 50 years reported use of a psychiatric medication, while around $30 \%$ of females over 60 years reported a psychiatric medication. ${ }^{4}$ Females had higher rates of psychiatric medication users after 15 to 20 years related to a combination of changes in users within and between medication categories.

Clear patterns of medication users were identified for most medication categories by age and sex through the course of the study, which likely represents some combination of acceptance of medication, symptom burden, and differences in access to care. Patterns of use for antidepressants for both males and females showed higher rates compared with earlier time points particularly among individuals between 15 to 35 and $>50$ years of age. Rates of benzodiazepine users increased with age from 10 to 60 years of age with some attenuation in most years following 60 years. Importantly, a notable decline in the rate of antipsychotic users was identified among individuals $<20$ years, which was particularly apparent among males from 2015 to 2018. This decline was likely secondary to changes in recommendations. ${ }^{10}$ The rate of ADHD medication users peaked in the teenage years with a rapid drop 
Figure 6. Proportion of Individuals Reporting Any Attention Deficit Hyperactivity Disorder Medication by Age and Sex. The figure represents the proportion of individuals reporting any attention deficit hyperactivity disorder medication during a year. Separate multivariable logistic regression used by sex and year category. Restricted cubic splines were utilized to model age. The dashed lines represented $95 \%$ confidence intervals.

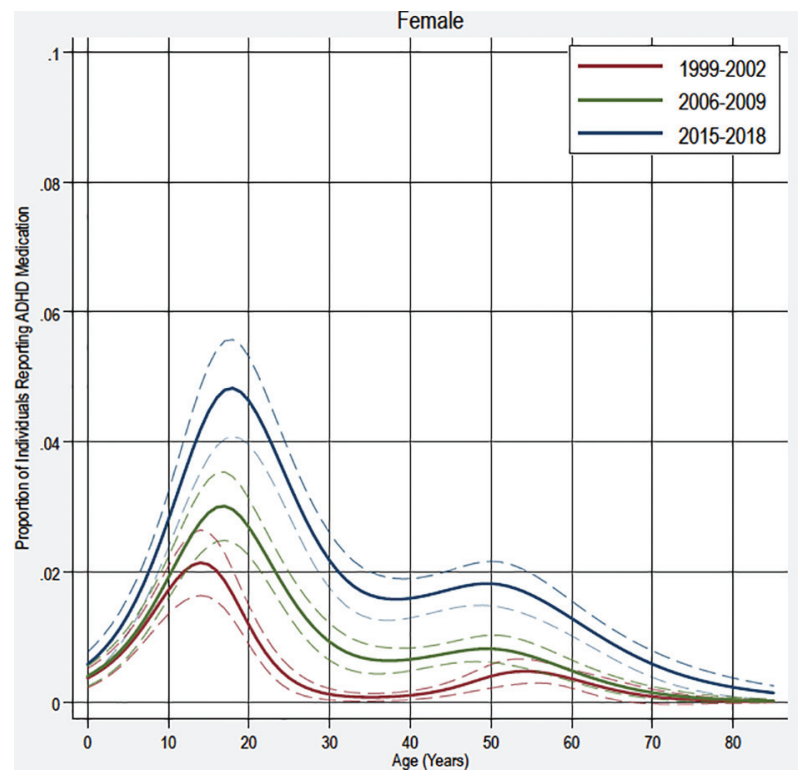

after adolescence. The most notable changes were the increase of the curve during teenage years as well as higher rates of medication users between 25 to 50 years throughout the study. These findings

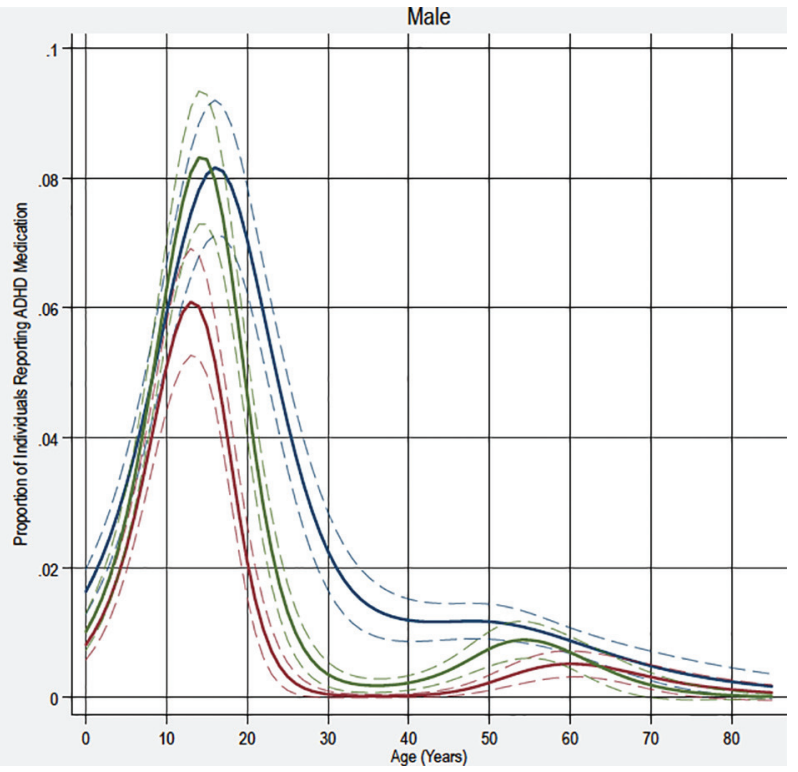

support both inter-and intragenerational changes in ADHD medication users.

The patterns of use for the most recent period (2015 to 2018) are visualized in Figure 7 and Figure

Figure 7. Proportion of Individuals Reporting Medication Use by Age and Sex in 2015 to 2018. The figure represents the proportion of individuals reporting use of different medications during a year between 2015 to 2018 . Separate multivariable logistic regression used by sex and medication category. Restricted cubic splines were utilized to model age.
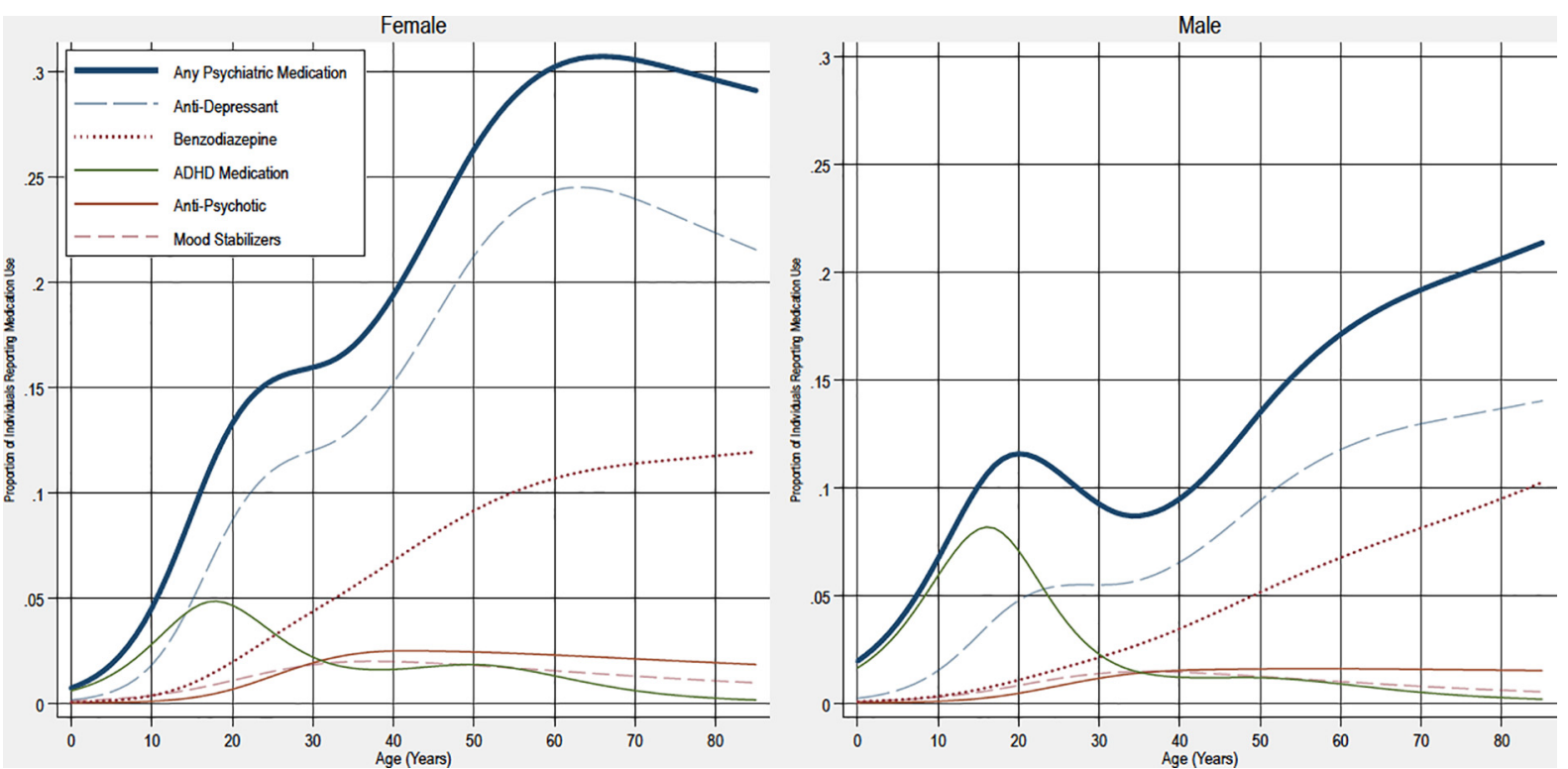
Figure 8. Female-To-Male Ratio of Medication Users by Life Decade, 2015 to 2018. Presents the rate ratio of female-to-male medication users by decade of life from 2015 to 2018. Benzodiazepines, mood stabilizers, and antipsychotic categories were coded for 0 to 19 years because of small sample size. Attention deficit hyperactivity disorder (ADHD) medications were top-coded at greater than 50 years because of sample sizes above this. Mood stabilizers and antipsychotics were top-coded at 60 years because of small sample size above this age.

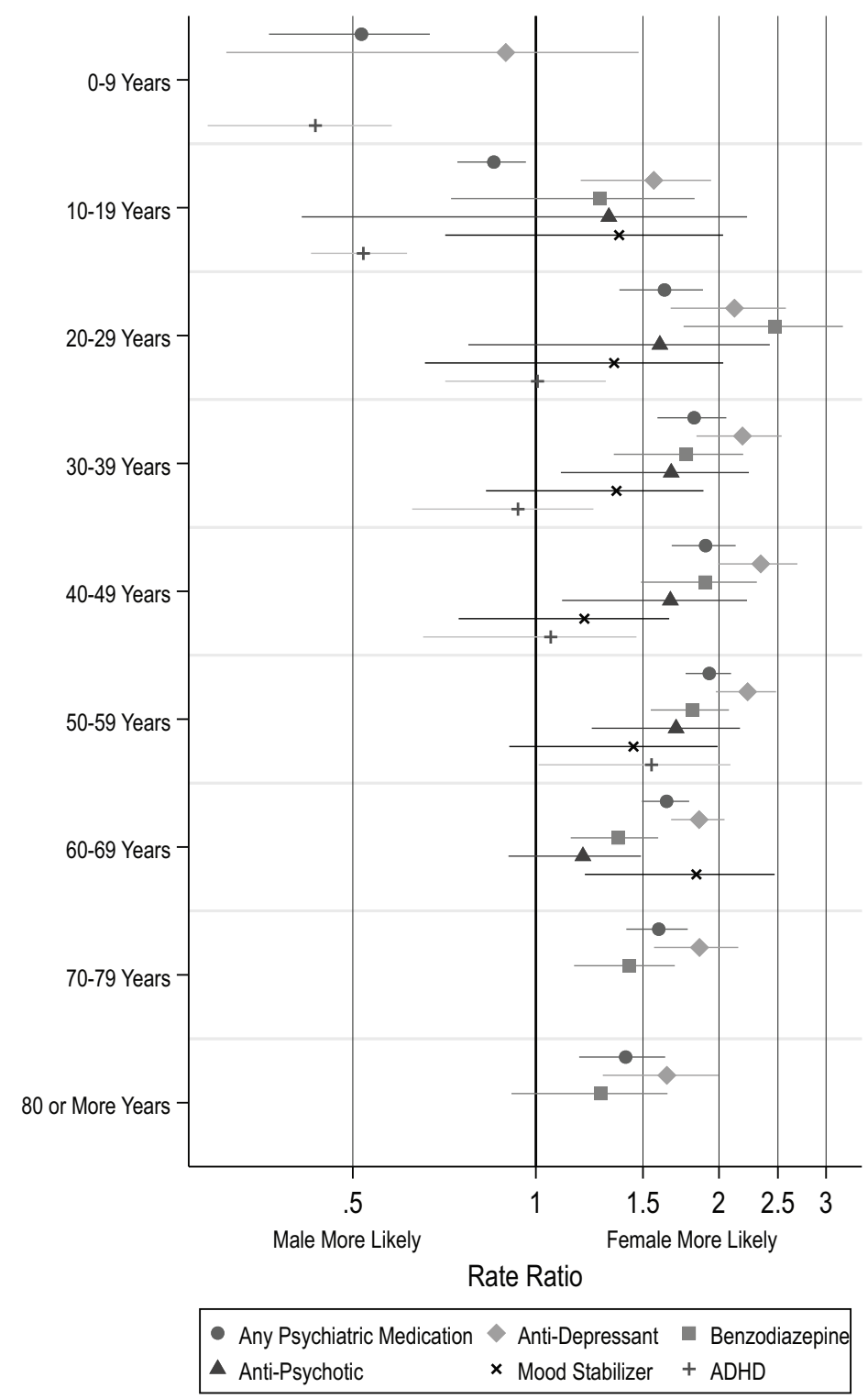

8 The comparative rate of users for males and females had a relatively consistent pattern for antidepressants, benzodiazepines, and antipsychotics. However, the age when females were clearly more likely to be medication users in these categories varied by age. There was no clear difference in the rate of mood stabilizer users by sex at any age, while ADHD medication had a unique pattern among the examined medication categories.
These data raise deeper questions about the meaning and importance of rates of psychiatric medication users. Different people will likely have wildly different interpretations of the importance of these data. Some will proclaim that advancements in medical science (ie, medications) have improved the quality of life of millions, while others will see these data as evidence of intrinsic flaws in modern society. These debates will likely 
continue. These data raise more questions such as: why the rates of psychiatric medication users are as high as they are when the evidence surrounding most of these medications shows modest short-term effect sizes with meager long-term data? ${ }^{11-15}$

The study had numerous limitations. First, medication use is self-reported (and confirmed through pharmacies). ${ }^{16}$ Previous studies have found chronic medications are well reported, but short-term medications (ie, benzodiazepines or trazodone) might be less so. ${ }^{17}$ Second, 4-year periods were selected to compare, which could fail to recognize important changes between or within these time periods. Third, the use of restricted cubic splines could overfit changes in age. Fourth, many of these medications could be used for nonpsychiatric purposes, and, therefore, this study should be interpreted as such. Fifth, the mood stabilizer category is not well defined as these medications have different mechanisms of action. Sixth, age had to be categorized for the comparison rates of female-to-male due to difficulty in calculating confidence intervals with survey-weighted data using splines. Seventh, the composition of the population at different age ranges could be different over time. For instance, individuals with bipolar disorder and schizophrenia are known to have notably shorter life spans than individuals without these conditions. ${ }^{18-20}$ In addition, if life expectancy of these conditions has changed over the last few decades, findings from this study could be impacted. Eighth, consideration should be given to the changing racial and ethnic makeup of the United Stated during the study periods.

\section{Conclusion}

The rates of individuals reporting use of psychiatric medications have increased over the last couple of decades. Rates and patterns of medication users have large variations by medication category, age, and sex. Patterns of use were stable for most medication categories.

I would like to thank Raj Mehta, MD for comments on the manuscript.

To see this article online, please go to: http://jabfm.org/content/ 34/4/732.full.

\section{References}

1. Moore TJ, Mattison DR. Adult utilization of psychiatric drugs and differences by sex, age, and race. JAMA Intern Med 2017;177:274-5.

2. Paulose-Ram R, Safran MA, Jonas BS, Gu Q, Orwig D. Trends in psychotropic medication use among U.S. adults. Pharmacoepidem Drug Safe 2007;16:560-70.

3. Paulose-Ram R, Jonas BS, Orwig D, Safran MA. Prescription psychotropic medication use among the U.S. adult population: results from the third National Health and Nutrition Examination Survey, 1988-1994. J Clin Epidemiol 2004;57: 309-17.

4. Brody DJ, Gu Q. Antidepressant use among adults: United States, 2015-2018. NCHS Data Brief, no. 377. Hyattsville, MD: National Center for Health Statistics. 2020.

5. Olfson M, King M, Schoenbaum M. Antipsychotic treatment of adults in the United States. J Clin Psychiatry 2015;76:1346-53.

6. Johansen ME, Niforatos JD. Benzodiazepine use in the USA is driven by long-term users: a repeated cross-sectional study of MEPS 2002-2016. J Gen Intern Med 2019;34:2717-9.

7. Jonas BS, Gu Q, Albertorio-Diaz JR. Psychotropic medication use among adolescents: United States, 2005-2010. NCHS Data Brief, no. 135. Hyattsville, MD: National Center for Health Statistics. 2013.

8. Olfson M, King M, Schoenbaum M. Stimulant treatment of young people in the United States. J Child Adolesc Psychopharmacol 2016;26:520-6.

9. Medical Expenditure Panel Survey. MEPS HC-209: 2018 Consolidated Data File. https://meps.ahrq.gov/ data_stats/download_data/pufs/h209/h209doc.pdf. Accessed October 12, 2020.

10. American Psychiatric Association; Choosing Wisely. https://www.choosingwisely.org/clinician-lists/ american-psychiatric-association-antipsychotics-inchildren-or-adolescents/. Accessed October 12, 2020.

11. Cipriani A, Zhou X, Del Giovane C, et al. Comparative efficacy and tolerability of antidepressants for major depressive disorder in children and adolescents: a network meta-analysis. Lancet 2016;388: 881-90.

12. Cipriani A, Furukawa TA, Salanti G, et al. Comparative efficacy and acceptability of 21 antidepressant drugs for the acute treatment of adults with major depressive disorder: a systematic review and network meta-analysis. Lancet 2018;391:1357-66.

13. Huhn M, Nikolakopoulou A, Schneider-Thoma J, et al. Comparative efficacy and tolerability of 32 oral antipsychotics for the acute treatment of adults with multi-episode schizophrenia: a systematic review and network meta-analysis [published correction appears in Lancet. 2019 Sep 14;394 (10202):918. Lancet 2019;394:939-51. 
14. Holbrook AM, Crowther R, Lotter A, Cheng C, King D. Meta-analysis of benzodiazepine use in the treatment of insomnia. CMAJ 2000;162:225-33.

15. Butler M, Urosevic S, Desai P, et al. Treatment for Bipolar Disorder in Adults: A Systematic Review. Comparative Effectiveness Review No. 208. (Prepared by the Minnesota Evidence-based Practice Center under Contract No. 290-201200016-I.) AHRQ Publication No. 18-EHC012EF. Rockville, MD: Agency for Healthcare Research and Quality; August 2018.

16. Medical Expenditure Panel Survey. MEPS HC206A: 2018 Prescribed Medicines https://meps.ahrq. gov/data_stats/download_data/pufs/h206a/h206adoc. pdf. Accessed October 11, 2020.
17. Hill SC, Zuvekas SH, Zodet MW. Implications of the accuracy of MEPS prescription drug data for health services research. Inquiry 2011;48:242-59.

18. Kessing LV, Vradi E, Andersen PK. Life expectancy in bipolar disorder. Bipolar Disord 2015;17:543-8.

19. Crump C, Sundquist K, Winkleby MA, Sundquist J. Comorbidities and mortality in bipolar disorder: a Swedish national cohort study. JAMA Psychiatry 2013;70:931-9.

20. Gale CR, Batty GD, Osborn DPJ, Tynelius P, Whitley E, Rasmussen F. Association of mental disorders in early adulthood and later psychiatric hospital admissions and mortality in a cohort study of more than 1 million men. Arch Gen Psychiatry 2012;69:823-31. 